reaction leaving a ${ }^{17} \mathrm{O}$ ion in its $\frac{1}{2}+$ state at $0.871 \mathrm{MeV}$.

This process can take place in at least two ways. First, the energy of the interaction can simply promote the odd neutron in ${ }^{17} \mathrm{O}$ from its ground state orbit to the one corresponding to the $0.871-\mathrm{MeV}$ state; this is the normal inelastic scattering mechanism. But an experimentally indistinguishable result is obtained if the odd neutron in the incident ${ }^{17} \mathrm{O}$ ion is transferred to the orbit in the target ${ }^{16} \mathrm{O}$ that leaves the resulting ${ }^{17} \mathrm{O}$ nucleus in its $0.851-\mathrm{MeV}$ state. This process gives a cross section that is peaked in the backward direction whereas the former process gives one peaked in the forward direction. At the energies of this experiment both amplitudes have broad angular peaks and similar absolute magnitudes, so significant interference effects can occur.

These cross sections were calculated using the distorted wave theory and the results obtained for the dominant transfer inelastic process above are shown in Fig. 1. The overall behaviour of the cross sections is given quite well but close examination shows fine structure in the data that is not reproduced theoretically. Additional calculations were then made including the contribution from the direct inelastic scattering process, and the results (Fig. 2) show that the fine structure is at least qualitatively reproduced. A perfect fit is not to be expected because higher order processes are not taken into account explicitly in this work. Some implicit account was taken of them by multiplying the direct inelastic scattering amplitude by a factor of $1.8 \mathrm{i}$ and this gives a much improved fit.

This work provides convincing evidence for the presence of interference

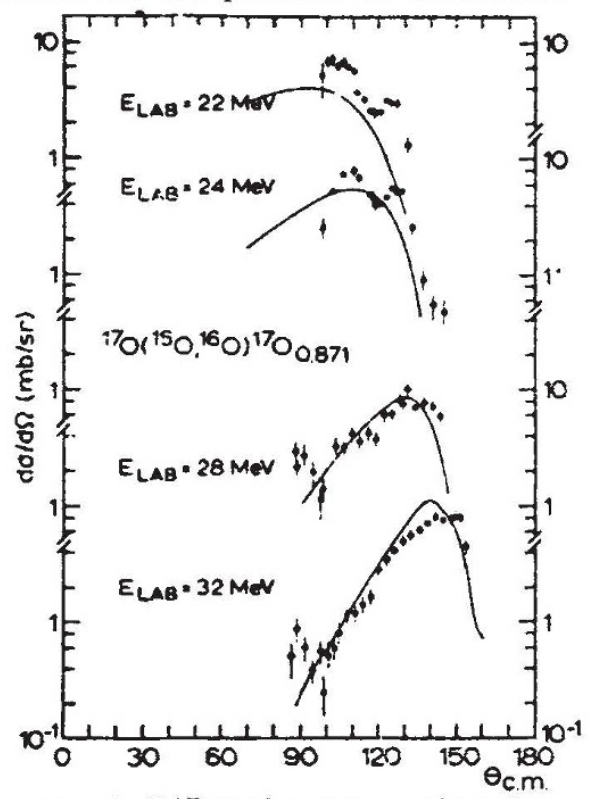

Fig. 1 Differential cross sections for the inelastic scattering of ${ }^{16} \mathrm{O}$ by ${ }^{17} \mathrm{O}$ compared with distorted wave calculations of the one-neutron transfer process.

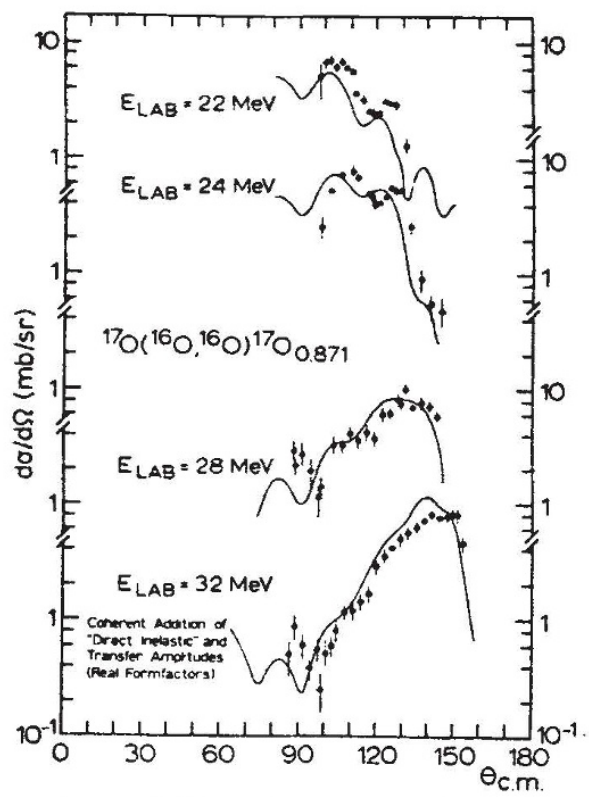

Fig. 2 Differential cross sections for the inelastic scattering of ${ }^{16} \mathrm{O}$ by ${ }^{17} \mathrm{O}$ compared with distorted wave calculations of the coherent addition of the direct inelastic and inelastic transfer process.

effects in the inelastic scattering of heavy ions and is likely to develop into a useful spectroscopic tool.

\section{Gene isolation and HnRNA}

\section{from a Correspondent}

HNRNA, an abbreviation for the more cumbersome 'heterogeneous nuclear RNA', has been a difficult, yet intrig. uing, class of RNA molecules to study. Although it was discovered in 1961, clear and formal evidence that HnRNA is a high molecular weight intermediate, or precursor, in the biosynthesis of messenger RNA has been discovered only recently. The turning point came (as J. Darnell of Columbia University, New York, explained at a symposium of the nucleotide group of the British Chemical and Biochemical Societies held in Glasgow on April 1 and 2) when it was found that both HnRNA and mRNA have the common and distinctive chemical feature of an extensive polyadenylic acid region at one end of the molecule.

These studies were made initially on the complex mixtures of both messenger RNA and of HnRNA present in a widely used human tumour cell line of epithelial origin (HeLa cells). The same type of precursor-product relationship has, however, been convincingly shown by both $\mathrm{R}$. Williamson (Beatson Institute, Glasgow) and K. Scherrer (Institute for Cancer Research, Lausanne) for the biosynthesis of the specific globin messenger RNAs, in the specialised red blood forming cells. Scherrer emphasised the complexity of the maturation process of the highest molecular weight HnRNA to the end product globin mRNA even in this well defined system. The biosynthesis of the heavy chain immunoglobulin mRNAs (A. R. Williamson, University of Glasgow) may be simpler as only two well defined sizes of HnRNA occur.

In the maturation of HnRNA at least $90 \%$ of the molecule is degraded in the nucleus. Both Darnell and R. H. Burdon (University of Glasgow) described uridylate-rich regions in this part of HnRNA. Furthermore, extensively repeated helical regions occur (Darnell).

The control of the synthesis of HnRNA is an area where it is easier to suggest mechanisms than to verify whether these mechanisms actually operate. R. S. Gilmour (Beatson Institute, Glasgow), however, is studying the induction of haemoglobin synthesis in chromatin (thus studying genes which are actively capable of being expressed) in a 'Friend' leukaemic cell, has isolated a mutant with more globin mRNA. This may be a 'transcriptional' mutant with an increased rate of synthesis of HnRNA. By contrast, the absence of $\alpha$-globin synthesis in a still-born child suffering from Hydrops foetalis (an extreme form of $\alpha$ thallasaemia) is caused by the absence of the $\alpha$-globin gene rather than by any fault in synthesis of mRNA (R. Williamson).

Two speakers, D. D. Brown (Carnegie Institute, Baltimore) and M. L. Birnstiel (University of Zurich) described the isolation and some properties of specific animal genes. Brown has made an extensive study of the reiterated genes coding for $5 \mathrm{~S}$ ribosomal RNA in the South African toad (Xenopus laevis) and he presented new evidence of the size and sequence heterogeneity of the 'spacer' regions which alternate with the coding regions in this extensively repeated gene. Furthermore, recent sequence studies on the spacer have shown that it consists in part of many short identical or nearly identical sequences in tandem, the average repeat length being sixteen bases.

The importance of the radioactlve sequence methodology was emphasised by F. Sanger (MRC Laboratory of Molecular Biology, Cambridge) when he described his recent work on sequencing DNA by radioactive methods. DNA may be labelled either 'directly' or by 'copying' methods. The first approach was used to derive two 50-long sequences in the bacteriophage $\Phi \mathrm{X}$; using the second approach he described the methods for extending a primer complimentary to the single stranded DNA of bacteriophage $\mathrm{f} 1$ using DNA polymerase to deduce a sequence of 81 residues. Undoubtedly the methods will be of very general application to many problems in molecular biology. 